\title{
Cytoreductive Surgery Followed by Hyperthermic Intraperitoneal Chemotherapy for Recurrent Ovarian Cancer with Incidental Bochdalek Hernia and Postoperative Bilateral Thalamic Infarct: A Case Report
}

\author{
Ilker Kahramanoglu ${ }^{a}$ Hasan Turan $^{a} \quad$ Ece Yamak Altinpulluk ${ }^{b}$ \\ Zahid Mammadov $^{\mathrm{a}}$ Tugan Bese ${ }^{\mathrm{a}}$ Macit Arvas $^{\mathrm{a}}$ Fuat Demirkiran $^{\mathrm{a}}$ \\ a Division of Gynecologic Oncology, Department of Obstetrics and Gynecology, Istanbul \\ University Cerrahpasa Medical Faculty, Istanbul, Turkey; ${ }^{b}$ Department of Anesthesiology \\ and Reanimation, Istanbul University Cerrahpasa Medical Faculty, Istanbul, Turkey
}

\section{Keywords}

Bochdalek hernia · Hyperthermic intraperitoneal chemotherapy · Thalamic infarction · Chest tube

\begin{abstract}
Congenital Bochdalek hernia is a defect of the diaphragm and very rare in adults. Only around 100 cases have been reported in the literature. Herein, we present a case with a recurrent ovarian cancer who underwent secondary cytoreductive surgery and hyperthermic intraperitoneal chemotherapy. An oval defect with dimensions of $3 \times 4 \mathrm{~cm}$ was seen in the left posterolateral site of the diaphragm during surgical exploration. In addition, a $6 \times 3 \mathrm{~cm}$ iatrogenic right-sided diaphragmatic defect was found and repaired. In the early postoperative period, a bilateral thalamic infarction occurred.




\section{Introduction}

Congenital Bochdalek hernia ( $\mathrm{CBH}$ ) develops as a result of the inappropriate fusion of the pleuroperitoneal fold and septum transversum at the site of the posterolateral lumbocostal trigone at the sixth week of gestation [1]. This entity was first described by McCauley in 1754 and then by Vincent Alexander Bochdalek in 1848. The incidence of this congenital defect is 1 in 2,500 births. Generally, the defect is left-sided (85\%) [1]. This type of diaphragmatic hernia is considered a perinatal disease that presents a few hours after birth, or in early infancy, and it is associated with significant morbidity and mortality [2]. $\mathrm{CBH}$ in adults is very rare; it remains asymptomatic until adulthood and generally presents with thoracic and/or abdominal symptoms aggravated in supine position [3].

We present a case of left-sided congenital Bochdalek hernia that remained asymptomatic and was discovered incidentally during secondary cytoreductive surgery for epithelial ovarian cancer; in this patient, a iatrogenic diaphragmatic defect was found, hyperthermic intraperitoneal chemotherapy (HIPEC) was performed and early postoperative bilateral anterior thalamic infarction developed.

\section{Case Report}

A 46-year-old female patient with a BMI of 25 was referred to our tertiary center (Cerrahpasa Medical Faculty, Gynecological Oncology Unit) in August 2016 with the diagnosis of platinum-resistant ovarian cancer. She had undergone primary cytoreductive surgery 6 months ago. Her medical history was unremarkable except for treatment for epithelial ovarian carcinoma. Positron emission tomography and computed tomography (CT) revealed implants on the right diaphragmatic surface after first-line adjuvant chemotherapy. She has never had any thoracic or abdominal symptoms and no history of major trauma. Initial preoperative evaluation of the patient revealed no significant sign of diaphragmatic hernia. Her physical examination was normal, including chest auscultation sounds and chest X-ray. Thoracic CT did not reveal any abnormal image that could be evaluated as a diaphragmatic hernia. Secondary cytoreductive surgery and HIPEC were planned. She was placed in flank position, and, under general anesthesia, laparotomy was performed. An oval defect with the dimensions of $3 \times 4 \mathrm{~cm}$ was seen in the left posterolateral site of the diaphragm (Fig. 1). The most distal portion of the inferior lobe of the left lung was visible through the defect during inspiration and expiration movements. There was no herniation or strangulation of abdominal components. In addition, the right diaphragm was completely stripped because of a tumor implant. Meanwhile, a $6 \times 3 \mathrm{~cm}$ iatrogenic diaphragmatic defect was detected (Fig. 2). Drainage chest tubes were inserted bilaterally via the 6th intercostal spaces to avoid leakage of the chemotherapeutic agents across repaired diaphragm and postoperative massive pleural effusion. The tissue around the congenital left-sided defect was excised circularly and the defect itself was repaired with interrupted nonabsorbable monofilament sutures. The iatrogenic defect was also repaired with interrupted nonabsorbable monofilament sutures. After complete cytoreduction had been achieved, hyperthermic intraperitoneal chemotherapy with doxorubicin $35 \mathrm{mg} / \mathrm{m}^{2}$ and paclitaxel $175 \mathrm{mg} / \mathrm{m}^{2}$ for $60 \mathrm{~min}$ at $42.5^{\circ} \mathrm{C}$ was delivered. No leakage via drainage tubes from the thoracic cavity was noted during the procedure. During the surgery, the patient was managed by standard monitoring, which involved continuous monitoring of electrocardiography, invasive blood pressure, central venous pressure, pulse oximetry, body temperature and hourly urine output. Arterial samples were drawn at 
regular periods for the determination of electrolytes, acid base status and hematocrit. Finally, the operation lasted $4 \mathrm{~h}$. At the end of the surgery, the patient remained intubated and was transferred to the intensive care unit for monitoring ongoing resuscitation along with fluid and electrolyte management. The patient was extubated $10 \mathrm{~h}$ after completion of surgery, and was transferred to the service on the 2 nd postoperative day. Prophylactic enoxaparin 4,000 IU was started $6 \mathrm{~h}$ after surgery. On the 3rd and 4th postoperative day, excessive sleepiness throughout the day and dyspraxia was noted. Bilateral anterior thalamic infarction caused by emboli that block the thalamosubthalamic artery was demonstrated on brain CT (Fig. 3). The dose of enoxaparin was changed from 4,000 IU once a day to 800 IU twice a day. The patient was discharged on the 12th day after surgery. The patient's symptoms improved within 3 weeks. Follow-up at 4 months after surgery showed no evidence of recurrence and no symptom regarding thalamic infarct.

\section{Discussion}

CBH generally presents in newborns with severe respiratory failure or in infancy due to herniation and/or strangulation of the abdominal contents [1]. The presentation of this defect in adulthood is rare. One of the best studies concerning the incidence of the defect in adults was carried out by Mullins et al. [4], who reviewed 13,138 CT scans. Their study revealed an incidence of $0.17 \%$, with $68 \%$ being right-sided defects and $77 \%$ of the patients being female [4]. Another study, done by Brown et al. [1], produced different results. This was ascribed to differences in the reviewed cases. Since the second study summarized symptomatic cases, demographics identified were different than those in asymptomatic ones [1]. This difference showed that more cases of right-sided CBH in females may remain silent than left-sided cases. These data were supported by several case reports [5]. Nevertheless, in our case, the defect was noted on the left side. Also, no herniation of intestinal loops was noted, and the patient has never had any thoracic or abdominal symptom that might be related to diaphragmatic hernia.

Diagnosis of the disease might be carried out by chest CT when there are clinically unexplained thoracic and abdominal symptoms. A chest CT can show the diaphragmatic defect and the herniated organs [6]. The chest CT of our patient did not reveal any of the abovementioned patterns. There are several cases concerning surgical repair of the defect - both laparoscopic, via laparotomy, and through the thoracic cavity [7-9]. We performed upper midline incision for laparotomy, and there was no need for another access to carry out the repair of the diaphragmatic defect. Repair of diaphragmatic defect can be performed by various techniques, using absorbable or nonabsorbable sutures in an interrupted or continuous fashion. From our point of view, surgeons may choose any of them provided that they are comfortable with the procedure.

Thoracic drainage using chest tubes may not always be necessary. The main reason for using bilateral chest tubes was to prevent massive pleural effusion due to HIPEC. In addition, intra-abdominal pressure increases during HIPEC, which may subsequently cause a cephalad shift of the diaphragm to increase peak airway pressures and reduce residual capacity. These changes may adversely affect oxygenation and cardiac output. Chest drains which were inserted prior to HIPEC reduce the increase in peak airway pressure, and by this way, the length of the intubation period and hospital stay can be decreased [10]. 
Postoperative bilateral thalamic infarct is a very rare complication of surgery. Malignancy itself, HIPEC, central venous cannulation, the position of the patient's neck, sudden blood loss and a long duration of the operation were noted as possible risk factors in our case [11].

In conclusion, although congenital Bochdalek hernia commonly occurs in the newborn and infancy period, it must not be excluded in the case of unexplained abdominal and/or thoracic symptoms in adults. Despite the fact that this kind of rare condition presents with pain, obstruction symptoms or pulmonary symptoms, it can be silent until discovered incidentally. Moreover, chest CT cannot always exclude this type of hernia. Iatrogenic diaphragmatic defects may occur in order to provide complete cytoreduction. HIPEC can be used after the diaphragmatic defects have been repaired. Thoracic tubes may be helpful to prevent pleural effusion. Secondly, cerebral embolism should be suspected in the presence of any neurological symptom in postoperative patients.

\section{Statement of Ethics}

Written informed consent was obtained from the patient for publication.

\section{Disclosure Statement}

The authors declare that they have no conflicts of interest.

\section{References}

1 Brown SR, Horton JD, Trivette E, Hofman LJ, Johnson JM: Bochdalek hernia in the adult: demographics, presentation, and surgical management. Hernia 2011;15:23-30.

-2 Greer JJ: Current concepts on the pathogenesis and etiology of congenital diaphragmatic hernia. Respir Physiol Neurobiol 2013;189:232-240.

- Z Zhou Y, Du H, Che G: Giant congenital diaphragmatic hernia in an adult. J Cardiothorac Surg 2014;9:31.

-4 Mullins ME, Stein J, Saini SS, Mueller PR: Prevalence of incidental Bochdalek's hernia in a large adult population. AJR Am J Roentgenol 2001;177:363-366.

5 Kocakusak A, Arikan S, Senturk 0, et al: Bochdalek's hernia in an adult with colon necrosis. Hernia 2005;9:284-287.

6 Mullins ME, Saini S: Imaging of incidental Bochdalek hernia. Semin Ultrasound CT MR 2005;26:28-36.

7 Tokumoto N, Tanabe K, Hideki Y, et al: Thoracoscopic-assisted repair of a Bochdalek hernia in an adult: a case report. J Med Case Rep 2010;4:366-370.

8 Suzuki T, Okamoto T, Hanyu K, et al: Repair of Bochdalek hernia in an adult complicated by abdominal compartment syndrome, gastropleural fistula and pleural empyema: report of a case. Int J Surg Case Rep 2014;5:82-85.

-9 Massloom HS: Acute bowel obstruction in a giant recurrent right Bochdalek's hernia: a report of complication on both sides of the diaphragm. N Am J Med Sci 2016;8:252-255.

-10 Schluermann CN, Hoeppner J, Benk C, Schmidt R, Loop T, Kalbhenn J: Intra-abdominal pressure, Cardiac Index and vascular resistance during hyperthermic intraperitoneal chemotherapy: a prospective observational study. Minerva Anestesiol 2016;82:160-169.

-11 Hawkes MA, Arena JE, Rollan C, Pujol-Vereis VA, Romero C, Ameriso SF: Bilateral paramedian thalamic infarction. Neurologist 2015;20:89-92. 


\section{Case Reports in Oncology}

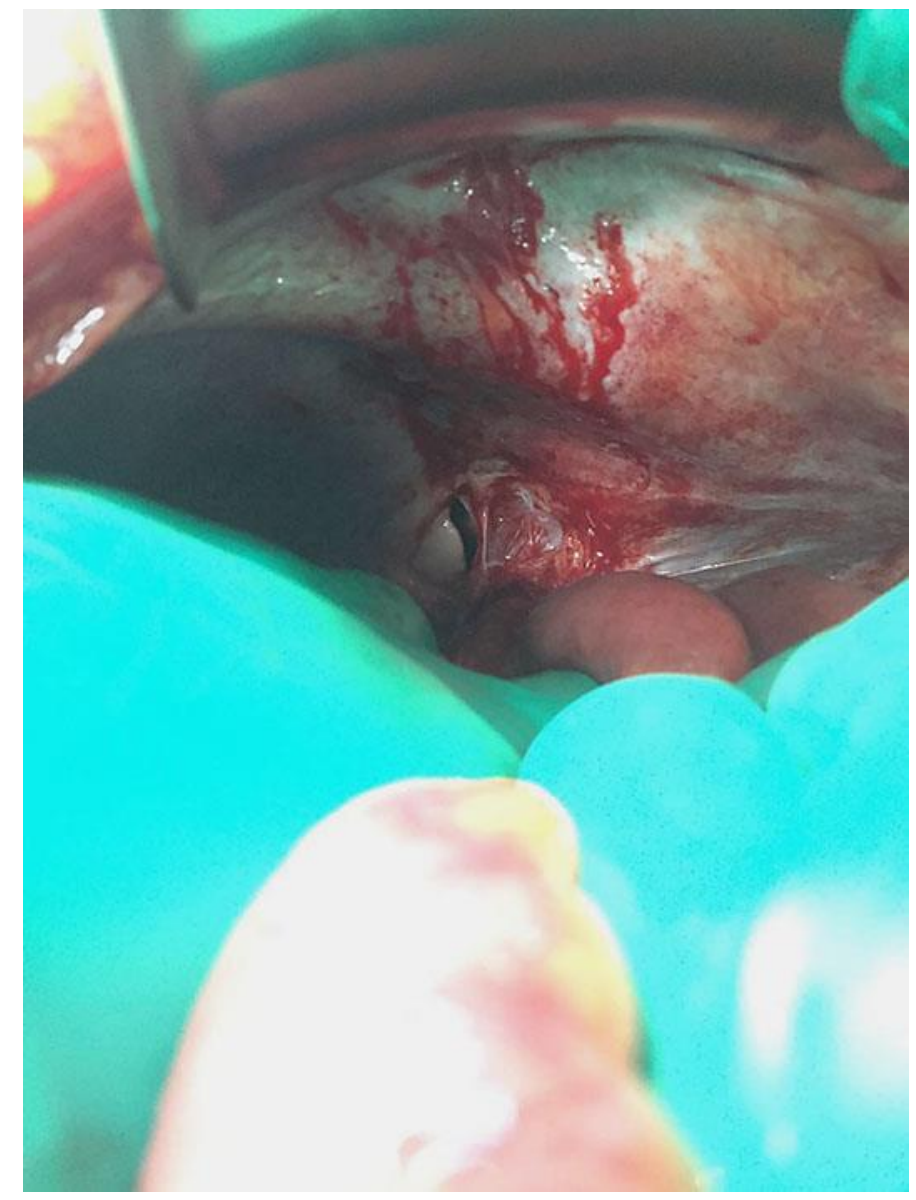

Fig. 1. Intraoperative photo of the left diaphragmatic defect.

Kahramanoglu et al.: Cytoreductive Surgery Followed by HIPEC for Recurrent Ovarian Cancer with $\mathrm{CBH}$ and Postoperative Bilateral Thalamic Infarct 


\section{Case Reports in Oncology}

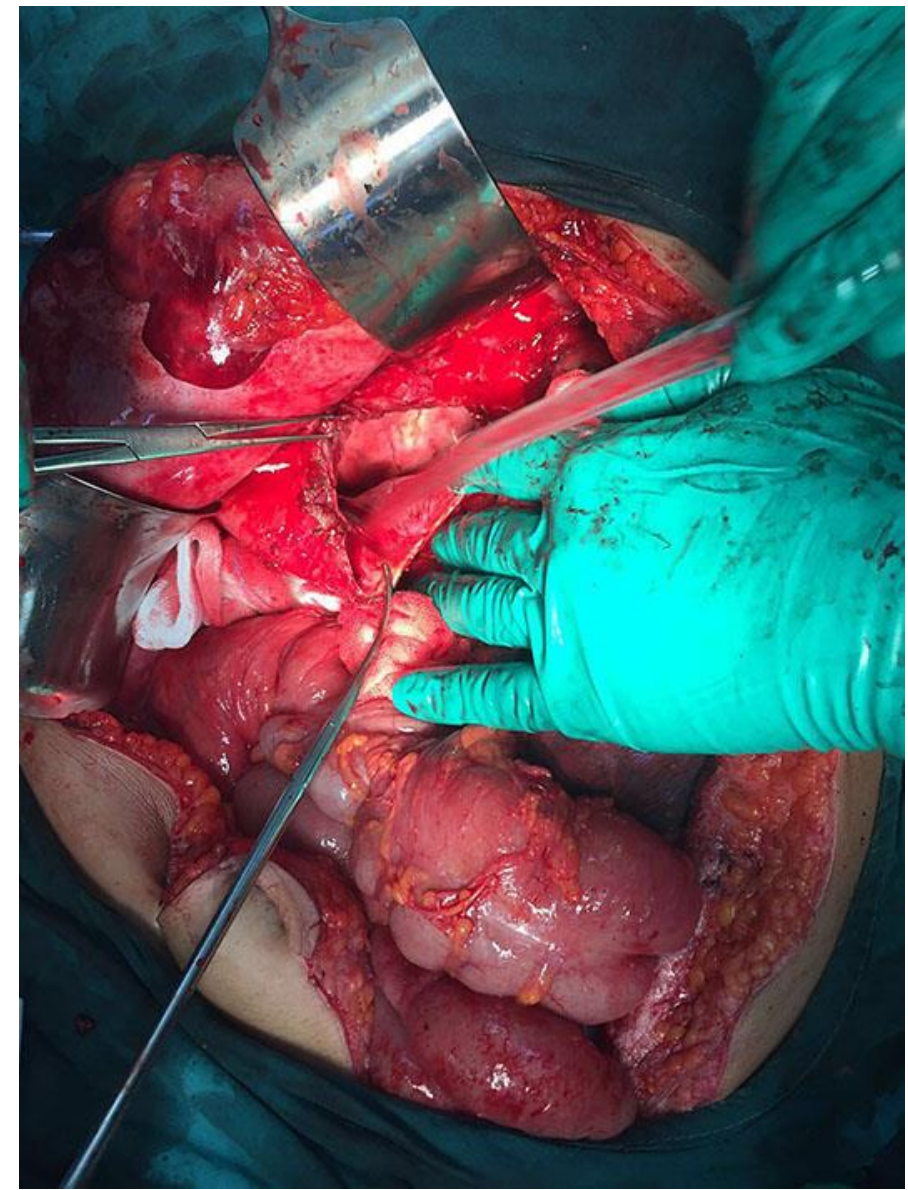

Kahramanoglu et al.: Cytoreductive Surgery Followed by HIPEC for Recurrent Ovarian Cancer with CBH and Postoperative Bilateral Thalamic Infarct

Fig. 2. Intraoperative photo of the right diaphragmatic injury. 


\section{Case Reports in Oncology}

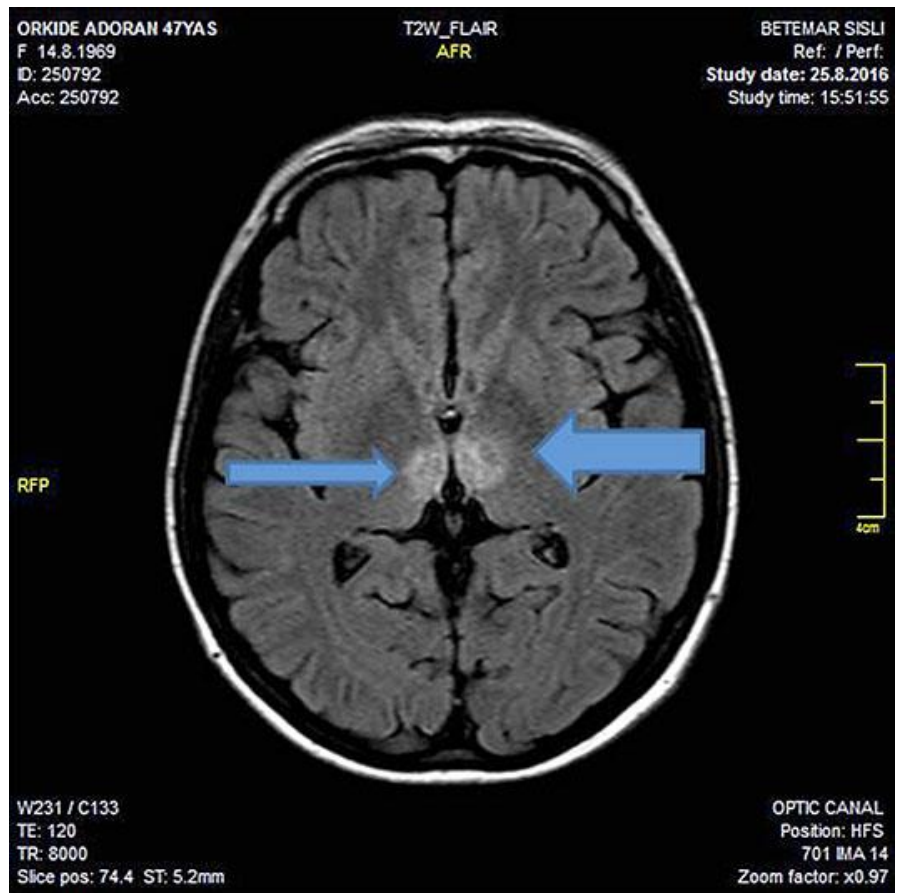

Fig. 3. An axial T2 FLAIR image (both arrows) shows bilateral subacute infarct of the thalamus.

Kahramanoglu et al.: Cytoreductive Surgery Followed by HIPEC for Recurrent Ovarian Cancer with CBH and Postoperative Bilateral Thalamic Infarct 